\title{
Editorial \\ Role of interleukin-7 in degenerative and inflammatory joint diseases
}

\author{
Joel AG van Roon and Floris PJG Lafeber
}

Department of Rheumatology and Clinical Immunology, University Medical Center Utrecht, Heidelberglaan, 3584 CX Utrecht, The Netherlands

Corresponding author: Joel AG van Roon, j.vanroon@umcutrecht.nl

Published: 18 April 2008

Arthritis Research \& Therapy 2008, 10:107 (doi:10.1186/ar2395)

This article is online at http://arthritis-research.com/content/10/2/107

(C) 2008 BioMed Central Ltd

See related research by Long et al., http://arthritis-research.com/content/10/1/R23

\begin{abstract}
IL-7 is known foremost for its immunostimulatory capacities, including potent $\mathrm{T}$ cell-dependent catabolic effects on bone. In joint diseases like rheumatoid arthritis and osteoarthritis, IL-7, via immune activation, can induce joint destruction. Now it has been demonstrated that increased IL-7 levels are produced by human articular chondrocytes of older individuals and osteoarthritis patients. IL-7 stimulates production of proteases by IL-7 receptorexpressing chondrocytes and enhances cartilage matrix degradation. This indicates that IL-7, indirectly via immune activation, but also by a direct action on cartilage, contributes to joint destruction in rheumatic diseases.
\end{abstract}

IL-7 is well-known for its strong immunostimulatory properties, in particular for the role it has in $\mathrm{T}$ and $\mathrm{B}$ cell homeostasis in mice and $\mathrm{T}$ cell homeostasis in humans. Less wellstudied is the role of IL-7 in (immuno)pathology, in particular its role in joint diseases. In the previous issue of Arthritis Research and Therapy, Long and colleagues [1] demonstrate that IL-7 protein is produced by articular chondrocytes. Production is increased upon stimulation with fibronectin fragments and a combination of $\mathrm{IL}-1$ and IL-6. Most interestingly, endogenous production of IL-7 by cartilage tissue is higher when obtained from older donors or from patients with osteoarthritis (OA). Through chondrocyte-expressed IL-7 receptor (IL-7R), this IL-7 is demonstrated to induce production of matrix metalloproteinase (MMP)-13 associated with enhanced release of proteoglycans from cartilage matrix. Thus, it has been suggested that IL-7 contributes in an autocrine manner to joint tissue destruction in $\mathrm{OA}$ and other joint diseases.

In support of a role for IL-7 in OA, it was recently shown that in synovial tissue of a substantial proportion of OA patients, IL-7 is expressed at a significant level (albeit lower than in rheumatoid arthritis patients) [2]. This IL-7 is considered to contribute to cartilage destruction indirectly through activation of inflammatory cells that secrete catabolic cartilagedestructive mediators, contributing to joint destruction. It has now been suggested that IL-7 is involved in cartilage destruction not only indirectly via inflammatory cells but also directly via IL-7R-expressing chondrocytes. However, although factors such as fibronectin fragment, and IL- 1 and IL-6 induce IL-7, the (patho)physiological triggers for IL-7 production by human articular chondrocytes in vivo remain to be determined. Mechanical stress is one of the mechanisms that should be considered. Definitive proof should be provided by blockade of the IL-7/IL-7R pathway, limiting intrinsic degenerative cartilage destruction in vitro and in vivo, preferably in experimental models of degenerative joint damage that mimic OA but with minor inflammation [3]. This is of particular importance since the amounts of IL-7 produced by chondrocytes in the experiments described by Long and colleagues are below the amounts needed to induce MMP-13 production and matrix degradation.

Irrespective of this, the data from the study of Long and colleagues underline the role of IL-7 in the induction of joint pathology in rheumatic diseases. It was recently demonstrated that IL-7, apart from its role in T cell development in humans, can stimulate inflammatory $T$ cells to produce tissue destructive cytokines that have a catabolic effect on cartilage and bone [4-7]. Together these studies suggest that IL-7 promotes joint destruction especially in patients that suffer from inflammatory (auto)immune diseases, many of which have increased IL-7 levels. Thus it was demonstrated that IL-7 induced $\mathrm{T}$ cell-dependent activation of monocytes/macrophages is associated, amongst other things, with tumour necrosis factor (TNF) $\alpha$ production [6]. Although it needs to be demonstrated that this results in joint damage in RA, the well-studied capacities of TNF $\alpha$ in this respect strongly

$\mathrm{IL}=$ interleukin; IL-R = IL receptor; MMP = matrix metalloproteinase; $\mathrm{OA}=$ osteoarthritis; $\mathrm{TNF}=$ tumour necrosis factor. 
suggest that this will be the case. TNF $\alpha$ is a potent inhibitor of cartilage matrix synthesis and an inducer of cartilage degradation (by activation of MMPs), processes that lead to loss of cartilage integrity. TNF $\alpha$ also activates fibroblasts to produce catabolic factors such as cytokines and MMPs that indirectly facilitate cartilage destruction. IL-7 has also recently been shown to induce $T$ cell-dependent osteoclast formation from monocytes. TNF $\alpha$ and RANKL (receptor activator of nuclear factor kappa B ligand) are crucial mediators in this IL7-driven osteoclast formation [7]. Interestingly, in the study of Long and colleagues, TNF $\alpha$ was not tested as an inducer of chondrocyte produced IL-7, nor did IL-7 stimulation lead to TNF $\alpha$ production by chondrocytes. This suggests that the chondrocyte IL-7/IL-7R pathway is independent from and additive with a TNF $\alpha$-driven pathway. This is supported by recent findings demonstrating TNF $\alpha$-independent IL-7-driven inflammatory and bone-destructive activity $[6,7]$.

IL-7 is also able to regulate joint pathology by $\mathrm{T}$ cell-driven immune activation in the absence of a clear inflammatory response. Experimental data have recently demonstrated the strong potential of IL-7 to facilitate bone loss. IL-7R-deficient mice display increased bone volume and bone density [8]. In contrast, IL-7-overexpressing transgenic mice are characterized by expanded bone marrow cavities with focal osteolysis of cortical bone and eroded bone surfaces [9]. In addition, estrogen deficient mice (induced by ovariectomy) are characterized by increased IL-7-driven T cell-dependent bone loss [10].

By giving a first glimpse of the direct effects of IL-7 on chondrocytes, the study of Long and colleagues contributes to our knowledge on the broad range of IL-7/IL-7R-driven pathways. In addition to its role in inflammation driven joint destruction, and its potential role in T cell-driven bone loss in the absence of prominent inflammation, direct harmful effects on cartilage can be added to the list of catabolic properties of IL-7. In this respect, the IL-7/IL-7R-stimulated pathology is a target of interest for the treatment of rheumatic diseases such as rheumatoid arthritis, osteoporosis and OA.

\section{Competing interests}

The authors declare that they have no competing interests.

\section{References}

1. Long DL, Blake S, Song XY, Lark M, Loeser RF: Human articular chondrocytes produce IL-7 and respond to IL-7 with increased production of matrix metalloproteinase-13. Arthritis Res Ther 2008, 10:R23.

2. Hartgring SA, Wenting MJ, Jacobs KM, Bijlsma JW, Lafeber FP, van Roon JA: IL-7-induced immune activation due to elevated expression of the IL-7 receptor in RA joints can be inhibited by soluble human IL-7 receptor. Arthritis Rheum 2007, 56:1991.

3. Marijnissen AC, van Roermund PM, TeKoppele JM, Bijlsma JW, Lafeber FP: The canine 'groove' model, compared with the ACLT model of osteoarthritis. Osteoarthritis Cartilage 2002, 10: 145-155.

4. Hartgring SA, Bijlsma JW, Lafeber FP, van Roon JA: Interleukin-7 induced immunopathology in arthritis. Ann Rheum Dis 2006, 65(Suppl 3):iii69-iii74.
5. van Roon JA, Verweij MC, Wijk MW, Jacobs KM, Bijlsma JW, Lafeber FP: Increased intraarticular interleukin-7 in rheumatoid arthritis patients stimulates cell contact-dependent activation of CD4(+) T cells and macrophages. Arthritis Rheum 2005, 52:1700-1710

6. van Roon JA, Hartgring SA, Wenting-van Wijk M, Jacobs KM, Tak PP, Bijlsma JW, Lafeber FP: Persistence of IL-7 activity and IL-7 levels upon TNF $\alpha$ blockade in patients with rheumatoid arthritis. Ann Rheum Dis 2007, 66:664-669.

7. Weitzmann MN, Cenci S, Rifas L, Brown C, Pacifici R: Interleukin-7 stimulates osteoclast formation by up-regulating the T-cell production of soluble osteoclastogenic cytokines. Blood 2000, 96:1873-1878.

8. Miyaura $C$, Onoe $Y$, Inada M, Maki $K$, Ikuta $K$, Ito M, Suda T: Increased B-lymphopoiesis by interleukin 7 induces bone loss in mice with intact ovarian function: similarity to estrogen deficiency. Proc Natl Acad Sci USA 1997, 94:9360-9365.

9. Valenzona HO, Pointer R, Ceredig R, Osmond DG: Prelymphomatous $B$ cell hyperplasia in the bone marrow of interleukin-7 transgenic mice: precursor B cell dynamics, microenvironmental organization and osteolysis. Exp Hematol 1996, 24:1521-1529.

10. Weitzmann MN, Pacifici R: Estrogen deficiency and bone loss: an inflammatory tale. J Clin Invest 2006, 116:1186-1194. 